\title{
A MULTISCALE APPROACH TO FRICTION ON SURFACES OF POLYMERIC SOLIDS AND IN BULK POLYMERIC MATERIALS
}

\author{
A. ZMITROWICZ ${ }^{1}$ \\ ${ }^{1}$ Institute of Fluid-Flow Machinery, Polish Academy of Sciences \\ ul. J. Fiszera 14, 80-231 Gdansk, Poland \\ azmit@imp.gda.pl
}

Key words: Anisotropic Dry Friction, Heterogeneous Dry Friction, Pin-on-disc Tests, Langevin Motion Equation, Anisotropic Viscous Friction, Macromolecule Friction

\begin{abstract}
Friction models of polymers must be considered in terms of specific microstructures, specific friction mechanisms and different scales on external surfaces of polymeric solids and inside the polymeric materials. Presented friction models take into account: evolution of surface microstructures, friction anisotropy and heterogeneity on surfaces of polymeric solids, micromechanical models of macromolecules, friction anisotropy in bulk polymeric materials
\end{abstract}

\section{INTRODUCTION}

Variations of dry friction with respect to a sliding direction and with respect to a position on a sliding trajectory have been observed on surfaces of polymeric solids, i.e. in polymer composites, reinforced elastomers, semi-crystalline polymers, self-assembled polymers and in polymers with an oriented surface roughness $[1,3,4]$. In some polymers, kinematics of sliding and friction initiate reorientation of macromolecules in the surface and near-surface material, and friction changes are observed $[1,3]$. In this study, the following quantities are taken as independent variables of friction models: unit vectors and oriented angles of sliding velocities, curvatures and unit normal vectors of sliding trajectories. The variables are used in constitutive equations of anisotropic and heterogoneus dry friction. In the frame of continuum thermodynamics, forms and variables of friction constitutive equations are restricted by the principle of objectivity and the Second Law of Thermodynamics. In pin-on-disc experiments $[1,3]$ the sliding trajectories of the polymer pin have been investigated in the form: concentric circles (PTFE, HDPE), Archimedean spiral and lemniscate of Bernoulli (UHMWPE). Movements of the polymer pin in pin-on-disc tests are analysed theoretically including friction heterogeneity.

Usually macromolecules in the bulk polymeric materials are randomly distributed, but under external loads and temperatures the orientation process of the macromolecules takes place. Polymers are modelled as assemblies of great number of isolated individual micro-elements composing the materials. In polymer melts, polymer solutions and gels, polymer liquid crystals and in self-assembled polymers, the micro-elements move with a highly anisotropic friction $[2,5]$. In stochastic dynamics, Langevin equation of motion describes Brownian movements of polymer chains with anisotropic friction [5]. In computational mechanics, various numerical methods are used for computing the macromolecule dynamics (MD, DEM, FEM). In this study, two approaches are applied for modelling of anisotropic friction in 


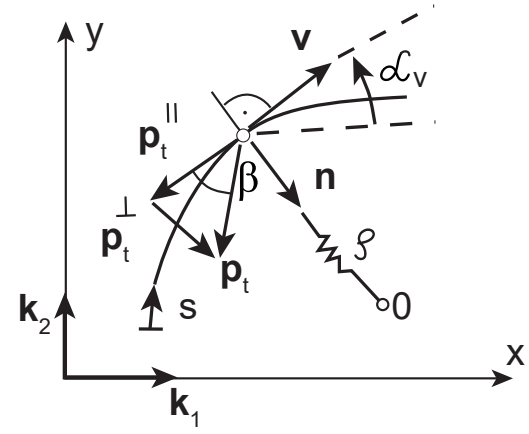

Figure 1: The sliding trajectory (s) of a material point in a plane; the friction force vector $\left(\mathbf{p}_{t}\right)$ with tangent and normal components $\left(\mathbf{p}_{t}^{\|}, \mathbf{p}_{t}^{\perp}\right)$, the angle of inclination $(\beta)$ and independent variables of the friction force models: the tangent and normal unit vectors $(\mathbf{v}, \mathbf{n})$, the oriented angle $\left(\alpha_{v}\right)$ and the radius of curvature $(\rho)$ of the sliding path

the bulk polymeric materials: (a) continuum based models, (b) micromechanical models. In the continuum approach, models of translational and rotational anisotropic viscous friction are investigated. In the micromechanical approach, kinematics and anisotropic friction are taken into account in sliding of bead-like macromolecules, rolling without or with slipping of rod-like macromolecules, spinning and sliding of disc-like macromolecules, snake-like sliding of long macromolecules. It is assumed that the micromechanical models of polymer macromolecules are in contact with a hypothetical plane in presence
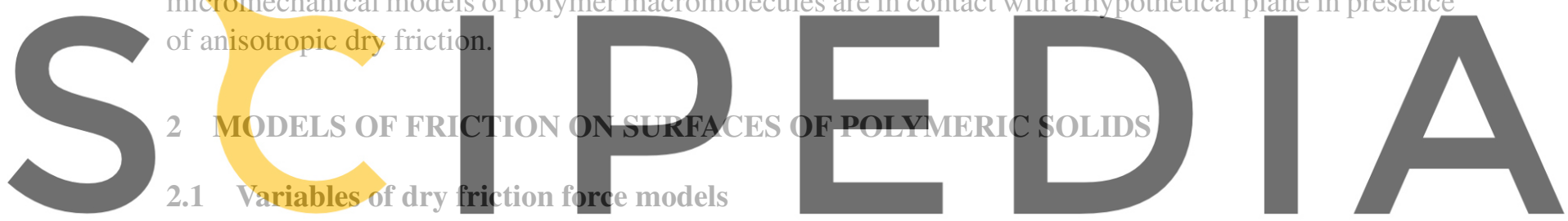

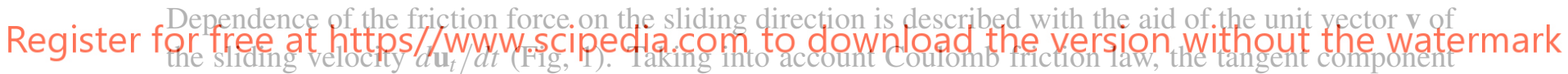
$\mathrm{p}_{t}$ of the contact force is defined as follows

$$
\begin{array}{rr}
\left|\mathbf{p}_{t}\right| \leq \mu\left|p_{n}\right| & \text { (stick) } \\
\mathbf{p}_{t}=-\mu\left|p_{n}\right| \mathbf{v},|\mathbf{v}|=1 \quad & \text { (slip) }
\end{array}
$$

where, $\mu$ is the friction coefficient, $p_{n}$ is the normal contact pressure (i.e. the normal component of the contact force). In the slip state the friction force equals $\mu\left|p_{n}\right|$ in magnitude, and is oppositely directed to the sliding velocity. Stick means the case that the sliding velocity remains zero. In the stick state, the tangent component of the contact force is a value between the positive and negative values of $\mu\left|p_{n}\right|$, opposite to the resultant force and equal to it. Wth the aid of the unit vector $\mathbf{v}$ as the independent variable, we extend Eq. (1) including friction anisotropy and heterogeneity [7, 8, 9, 10, 11, 12].

Asymmetric surface roughness can cause asymmetry of friction, i.e. variations in friction when one slides forward and then one slides backward, for instance, smooth forward sliding and rough backward sliding. There is no center of symmetry in this type of friction. The friction description must include the sense of the sliding direction. Due to this we introduce the oriented angle (Fig. 1) as the independent 
variable in friction equations. $\alpha_{v} \in<0,2 \pi>$ is the measure of the oriented angle between the reference direction in the contact surface (e.g. $O x$-axis, Fig. 1) and the sliding direction $\mathbf{v}[7,8]$.

Dependence of the friction force on the position in the sliding trajectory is described with the aid of the radius of curvature (Fig. 1). This is the case of friction heterogeneity $[9,10,11]$. According to the Frenet-Serret first formula, the first derivative of the unit vector $\mathbf{v}$ with respect to the parametrization $\mathrm{s}$ of the trajectory is given by

$$
\frac{d \mathbf{v}}{d s}=\frac{\mathbf{n}}{\rho},
$$

where, $s$ is the one-dimensional parameterization of the sliding path, $\rho$ is the radius of curvature of the sliding path, $\mathbf{n}$ is the unit vector normal to the sliding trajectory, $\mathbf{n} \cdot \mathbf{v}=0,|\mathbf{n}|=1$. Then, the friction force vector is defined as the following function

$$
\mathrm{p}_{t}=\mathrm{p}_{t}\left(p_{n}, \mathbf{v}, \frac{\mathbf{n}}{\rho}\right)=-\left|p_{n}\right| f\left(\mathrm{v}, \frac{\mathbf{n}}{\rho}\right) \text {. }
$$

\subsection{Dependence of the friction force on the sliding direction}

The following single-term polynomial with respect to the sliding velocity unit vector $\mathbf{v}$ is the linear model of anisotropic fiction [11]
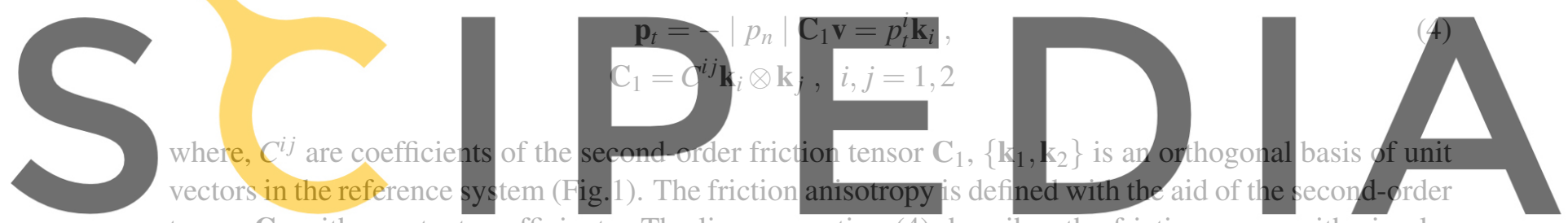

tensor $\mathbf{C}_{1}$ with constant coefficients. The linear equation (4) describes the friction cones with circular

\section{Register ford elliptical cross-sections. \\ or free at https//www.scipedia.com to download the version without the watermark}

The friction isotropy is illustrated by the friction cone with circular cross-section. The isotropic friction

tensor is the spherical tensor $\mathbf{C}_{1}=C^{11} \mathbb{1}$ with one coefficient, and its representation matrix is as follows

$$
\left[\mathbf{C}_{1}\right]=\left(\begin{array}{cc}
C^{11} & 0 \\
0 & C^{11}
\end{array}\right)
$$

The friction orthotropy is illustrated by the friction cone with elliptical cross-section. The orthotropic friction tensor is the symmetric tensor $\mathbf{C}_{1}=\mathbf{C}_{1}^{T}$, and its representation matrix has three coefficients

$$
\left[\mathbf{C}_{1}\right]=\left(\begin{array}{ll}
C^{11} & C^{12} \\
C^{12} & C^{22}
\end{array}\right)
$$

The following polynomial function of the sliding velocity unit vector $\mathbf{v}$ is the nonlinear model of anisotropic friction [11]

$$
\begin{array}{r}
\mathbf{p}_{t}=-\left|p_{n}\right|\left[\mathbf{C}_{1} \mathbf{v}+\mathbf{C}_{2}(\mathbf{v} \otimes \mathbf{v} \otimes \mathbf{v})+\ldots\right], \\
\mathbf{C}_{2}=C^{i j k l} \mathbf{k}_{i} \otimes \mathbf{k}_{j} \otimes \mathbf{k}_{k} \otimes \mathbf{k}_{l}, \quad i, j, k, l=1,2
\end{array}
$$


where, $\mathbf{C}_{2}$ is the fourth-order friction tensor. As independent variable is taken third-order tensor composed by the tensor products of the unit vectors $\mathbf{v}$. Other higher order friction tensors can be included in Eq. (7) [11]. The nonlinear equation (7) describes the friction cones with complex shapes of crosssections.This is important on external surfaces of crystals.

\subsection{Dependence of the friction force on the oriented angle}

In the case of non-centrosymmetric friction, the change of sense of the sliding direction must be included in anisotropy description. Therefore, the friction tensors in the linear and nonlinear friction equations can depend on the sliding direction and on the oriented angle, i.e. $\mathbf{C}_{i}\left(\boldsymbol{\alpha}_{v}\right)$, where, $\left.\boldsymbol{\alpha}_{v} \in<0,2 \pi\right\rangle$ is the measure of the oriented angle $[7,8]$. In this case the friction tensors have non-constant coefficients.

The friction tensors are assumed to be trigonometrical polynomials of the variable $\alpha_{v}$ as follows

$$
\mathbf{C}_{i}\left(\alpha_{v}\right)=\mathbf{C}_{i 0}+\mathbf{C}_{i 1} \cos \left(n_{i} \alpha_{v}\right)+\mathbf{C}_{i 2} \sin \left(m_{i} \alpha_{v}\right), \quad n_{i}, m_{i}=0,1,2,3, \ldots
$$

where, $\mathbf{C}_{i l}(i=1,2, \ldots, n ; l=0,1,2)$ are tensors with constant coefficients. If we restrict the equation (8) to the second-order friction tensors, then the friction force vector has the following form

$$
\mathbf{p}_{t}=-\left|p_{n}\right|\left[\mathbf{C}_{10}+\mathbf{C}_{11} \cos \left(n_{1} \alpha_{v}\right)+\mathbf{C}_{12} \sin \left(m_{1} \alpha_{v}\right)\right] \mathbf{v}
$$

If the parameters $n_{1}, m_{1}$ are odd numbers, then Eq. (9) describes non-centrosymmetric anisotropic friction. The tensors $\mathrm{C}$

2.4 Dependence of the friction

The constitutive equation of the heterogenous friction force
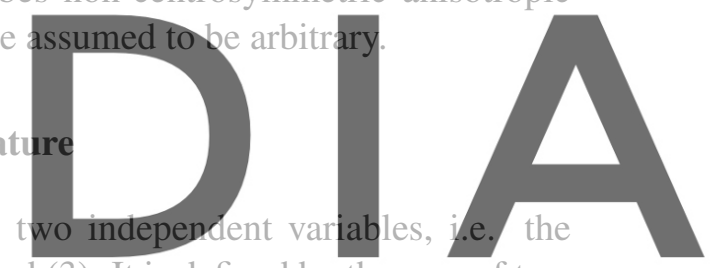

sliding velocity unit vector $\mathbf{v}$ and its derivative $\mathbf{d v} / \mathrm{ds}$, see Eqs (2) and (3). It is defined by the sum of two Register for fringe-term at phlynomials [9, 10, 11]

$$
\begin{array}{r}
\mathrm{p}_{t}(\rho)=-\left|p_{n}\right|\left(\mathbb{C}_{1} \mathbf{v}+\mathbb{E}_{1} \mathbf{n} \frac{1}{\rho}\right) \\
\mathbf{E}_{1}=E^{i j} \mathbf{e}_{i} \otimes \mathbf{e}_{j}, \quad\left\{\mathbf{e}_{1}, \mathbf{e}_{2}\right\} \equiv\{\mathbf{v}, \mathbf{n}\}, \quad i, j,=1,2
\end{array}
$$

Two second-order tensors $\mathbf{C}_{1}$ and $\mathbf{E}_{1}$ describe frictional anisotropy and heterogeneity effects induced by the sliding kinematics. The tensor $\mathbf{E}_{1}$ describes constraints imposed on the motion in the directions tangent and normal to the sliding path. These constraints are functions of the first power of the radii of curvature $\rho$ of the sliding path. The tensor $\mathbf{E}_{1}$ is defined with the aid of unit vectors tangent and normal to the sliding trajectory at the given point of the trajectory. The contraction of the tensor $\mathbf{E}_{1}$ and the vector of the independent variable $\mathbf{n} / \rho$ gives the following

$$
\mathbf{E}_{1} \frac{\mathbf{n}}{\rho}=\left(E^{11} \mathbf{v} \otimes \mathbf{v}+E^{12} \mathbf{v} \otimes \mathbf{n}+E^{21} \mathbf{n} \otimes \mathbf{v}+E^{22} \mathbf{n} \otimes \mathbf{n}\right) \frac{\mathbf{n}}{\rho}=\frac{E^{12}}{\rho} \mathbf{v}+\frac{E^{22}}{\rho} \mathbf{n}
$$

According to Eq. (11), the sliding path curvature generates: (a) dissipative type component i.e. an additional friction $\left(E^{12} / \rho\right)$ and (b) gyroscopic type component i.e. a sliding constraint $\left(E^{22} / \rho\right)$. In other 
words, $E^{12}$ is the coefficient of constraints imposed on the motion in the direction tangent to the sliding path, $E^{22}$ is the coefficient of constraints imposed on the motion in the direction normal to the sliding path.

Other higher order friction tensors can be included in Eq. (10), i.e. fourth-order tensor $\mathbf{C}_{2}$ see Eq. (7), and by analogy fourth-order tensor $\mathbf{E}_{2}$ [11]. In the case of $\mathbf{E}_{2}$ as idependent variable is taken third-order tensor composed by the tensor products of the unit vector $\mathbf{v}$ and $\mathbf{n} / \rho$. Eq. (10) with fourth-order tensors contains the radii of curvature $\rho$ of the sliding trajectory raised to higher powers.

\subsection{Thermodynamic restrictions of friction models}

The proposed friction equations satisfy the axiom of objectivity $[11,12]$. The material objectivity means that the friction force function Eq. (3) of the scalar $p_{n}$, the unit vector $\mathbf{v}$ and the vector $\mathbf{n} / \rho$ must be form-invariant with respect to any orthogonal transformation from the full orthogonal group $O, \mathbf{R}$ is the tensor of the orthogonal transformation, i.e.

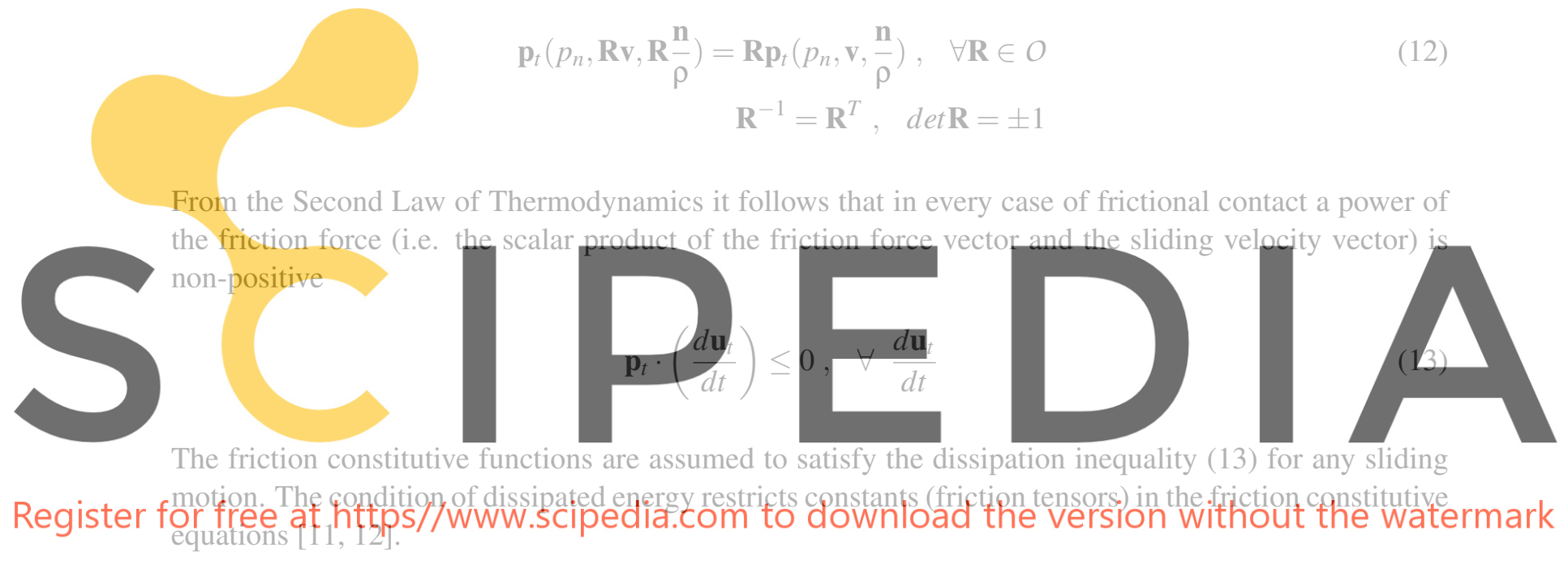

\subsection{Movements of the polymer pin in pin-on-disc tests}

In experiments $[1,3]$ the sliding trajectories of the polymer pin have been investigated in the form: concentric circles (PTFE, HDPE), Archimedean spiral and lemniscate of Bernoulli (UHMWPE). We analyse theoretically movements of the polymer pin in pin-on-disc tests taking into account friction heterogeneity .

Let us consider motion of a material point (the pin) in a plane with the given trajectory. The motion of the point in the refernce system $O x y$ is described by the following equation

$$
m \ddot{\mathbf{x}}=\mathbf{F}+\mathbf{p}_{t}
$$

where, $m$ is the mass of the material point, $\mathbf{x}$ is the position vector with respect to the reference system $O x y, \mathbf{p}_{t}$ is the friction force vector, $\mathbf{F}$ is the central force. The motion equation transformed to the local 
basis defined by the unit vectors tangent and normal to the sliding trajectory $\{\mathbf{v}, \mathbf{n}\}$ (Fig. 1) has the form as follows

$$
\begin{aligned}
& m \frac{d V}{d t}=F^{\|}+p_{t}^{\|} \\
& m \frac{V^{2}}{\rho}=F^{\perp}+p_{t}^{\perp}
\end{aligned}
$$

where, $V$ is the magnitude of the sliding velocity of the material point. The accelration vector $\ddot{\mathbf{x}}$ is replaced by tangent and centrifugal components. In Eq. (15) we need the tangent and normal components of the friction force vector with respect to the trajectory

$$
\mathbf{p}_{t}=p_{t}^{\|} \mathbf{v}+p_{t}^{\perp} \mathbf{n}
$$

As the sliding trajectory we assume a circle of the radius $R$ attached to the center of concentric circles. In polar coordinates $(R, \theta)$ the circle equation is given by the radius $R$ constant for any value of $\theta$, i.e.

$$
R(\theta)=R=\text { const }, \quad \theta \in<0,2 \pi>,
$$

The radius of curvature $\rho$ of the circle is constant
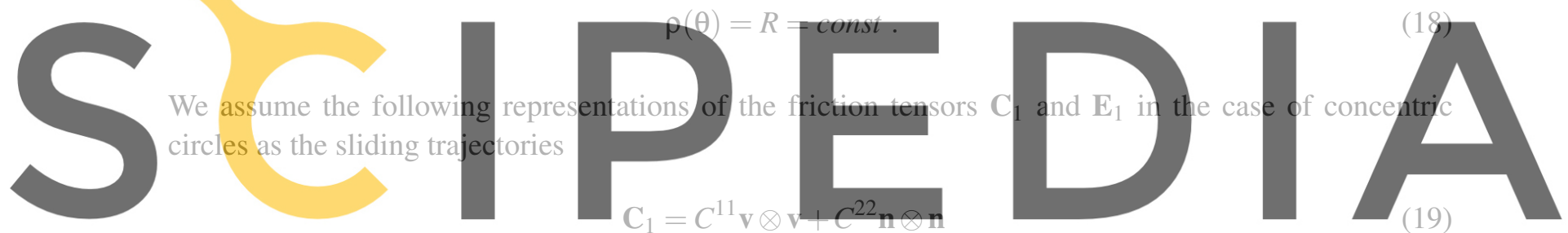

Register for free at https//www.scipedia.com to download the version without the watermark

where, $C^{11}$ defines friction along the circles, $C^{22}$ defines friction along the radii of the circles. The tangent and normal components of the friction force have the following form

$$
\mathbf{p}_{t}(R)=-\left|p_{n}\right|\left[\left(C^{11}+\frac{E^{12}}{R}\right) \mathbf{v}+\frac{E^{22}}{R} \mathbf{n}\right]=p_{t}^{\|} \mathbf{v}+p_{t}^{\perp} \mathbf{n}
$$

The curvature of the circular trajectory generates the dissipative type component, i.e. the additional friction $\left(E^{12} / R\right)$, and gyroscopic type component $\left(E^{22} / R\right)$.

Archimedean spiral specified as the sliding trajectory in polar coordinates $(R, \theta)$ is given by the radius $R$ as the linear function of the angle $\theta$

$$
R(\theta)=a \theta, \quad \theta \in<0, \infty), \quad a=\text { const }
$$

The radius of curvature $\rho$ of the Archimedean spiral is a monotonically increasing function of the angle $\theta$ as follows 


$$
\rho=\frac{a\left(\theta^{2}+1\right)^{3 / 2}}{\theta^{2}+2}
$$

In the case of of Archimedean spiral we have assumed isotropic second-order tensor $\mathbf{C}_{1}$ with one coefficient and orthotropic fourth-order tensor $\mathbf{C}_{2}$ with two coefficients [11]. Furtermore, we have included the tensor $\mathbf{E}_{1}$ with one coefficient and the tensor $\mathbf{E}_{2}$ with two coefficients [11]. The friction force vector for the sliding along the Archimedean spiral has the tangent (dissipative) and normal (gyroscopic) components with the radii of curvature raised to powers 1,2 and 3 [11].

Lemniscate of Bernoulli as the sliding trajectory is defined in polar coordinets by the radius $R$ as the trigonometrical function of the angle $\theta$

$$
R(\theta)= \pm a \sqrt{2 \cos (2 \theta)}, \quad a=\text { const }
$$

The radius of curvature of the lemniscate is also trigonometrical function

$$
\rho(\theta)=\frac{2 a^{2}}{3 R(\theta)}
$$

This is an oscilating function with respect to the angle $\theta$. The tangent and normal components of the friction force vector can be detrmined in the similar way as in the case of Archimedean spiral.
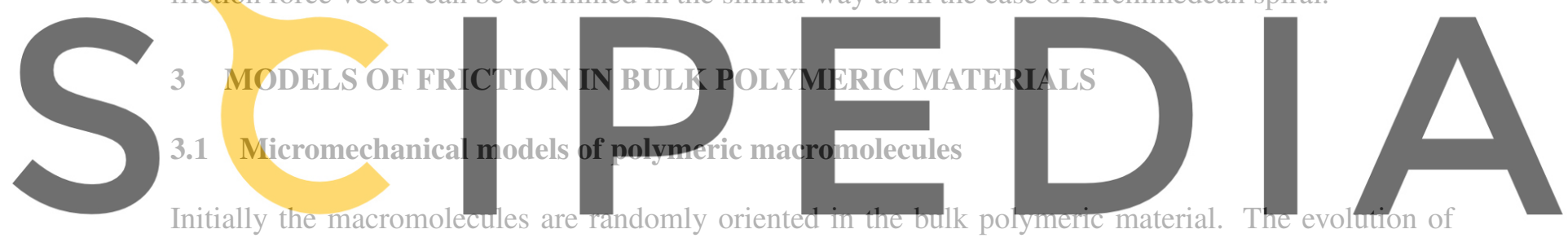

the macrostructure takes place under large loads (tension, shear), at high temperatures and under large

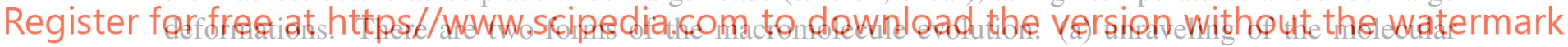

chains, (b) relative motion of the unraveled chains or their segments through the surrounding moving

molecules. The polymeric macromolecules are considered as independent kinematical elements. They have translational and rotational degrees of freedom [2, 5].

The macromolecules are modelled with the aid of idealized simple shapes. In the literature, the following classical models of the macromolecules are proposed: (a) Rouse model - the macromolecules are chains of spherical beads and springs (or rods), (b) De Gennes tube model - the long macromolecules move like a snake inside long and narrow tube, (c) rod-like and disc-like models of macromolecules in the case of polymers in liquid crystal phase. Nano-meter is the length scale of the polymer chains. Nano-second is the time scale in motion simulations.

\subsection{Brownian motions of polymer chains with anisotropic friction}

Polymer macromolecules move with respect to neighboring macromolecules with very high friction anisotropy [5]. Motion of the i-th bead (i.e. a segment of the polymer macromolecule) follows the Langevin equation: 


$$
\begin{array}{r}
m \frac{d^{2} \mathbf{r}_{i}}{d t^{2}}=\left[B^{11} \mathbf{k}_{1} \otimes \mathbf{k}_{1}+B^{22}\left(\mathbf{1}-\mathbf{k}_{1} \otimes \mathbf{k}_{1}\right)\right] \frac{d \mathbf{r}_{i}}{d t}-\mathbf{f}_{i}-\mathbf{F} \\
\mathbf{1}=\mathbf{k}_{1} \otimes \mathbf{k}_{1}+\mathbf{k}_{2} \otimes \mathbf{k}_{2}+\tilde{\mathbf{n}} \otimes \tilde{\mathbf{n}}, \quad B^{22} \gg B^{11}
\end{array}
$$

where, $m$ is the mass of the bead, $\mathbf{r}_{i}(t)$ is the bead coordinate (i.e. the position vector in $3 \mathrm{D}$ space), $\mathbf{f}_{i}$ is the stochastically fluctuating force (Gaussian white noise), $\mathbf{F}$ is other external force, $\mathbf{k}_{1}$ and $\mathbf{k}_{2}$ are the unit vectors tangent and normal to the particular chain segment in a horizontal plane, $\tilde{\mathbf{n}}$ is the unit vector in the transverse direction, $\mathbf{1}$ is the unit tensor, $B^{11}, B^{22}$ are friction coefficients for motions tangent and normal to the particular chain segment respectively (the lowest friction coefficient and the largest friction coefficient). Brownian dynamics simulations are the useful tool for study motions of polymer macromolecules and for prediction of polymer behaviours.

\subsection{Other computational methods in dynamics of polymer chains}

In computational mechanics MD, DEM and FEM are used for computing the macromolecule dynamics and for predicting properties of polymers. Molecular Dynamics is used for movements of systems of individual macromolecules. Discrete Element Method is used to simulate motions of small grains e.g. agglomerates and collectives of macromolecules. Finite Element Method has been applied for calculations of rode-like microstructures [2].

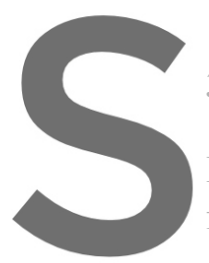

3.4 Continuum-based models of anisotropic viscous friction
In Langevin motion equation $(25)$ the friction force is the viscous
friction in Eq. (25) we extend taking the force of translational aniso

$$
\mathrm{p}=-\mathrm{B} \frac{\mathrm{du}}{\mathrm{d}}
$$
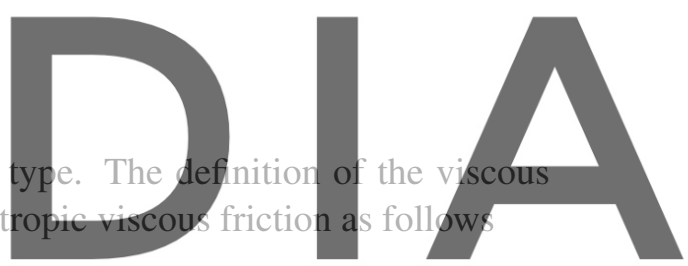

(26)

Register for free at https//www.scipedia.com to dlownload the version without the watermark where, $\mathbb{B}$ is the tensor of translational anisotropic viscous friction, $d \mathbf{u} / d t$ is the translational relative velocity

$$
\mathbf{B}=B^{i j} \mathbf{k}_{i} \otimes \mathbf{k}_{j}, \mathbf{k}_{3} \equiv \tilde{\mathbf{n}}, \quad i, j=1,2,3
$$

The vectors of the friction force $\mathbf{p}$ and the velocity $d \mathbf{u} / d t$ have horizontal and transversal components with respect to the flow direction

$$
\mathbf{p}=\mathbf{p}_{t}+\mathbf{p}_{n}, \quad \frac{d \mathbf{u}}{d t}=\frac{d \mathbf{u}_{t}}{d t}+\frac{d \mathbf{u}_{n}}{d t}
$$

In the case of horizontal isotropy and transversal isotropy, the representation matrix of the viscous friction tensor $\mathbf{B}$ has two coefficients as follows

$$
[\mathbf{B}]=\left(\begin{array}{ccc}
B^{11} & 0 & 0 \\
0 & B^{11} & 0 \\
0 & 0 & B^{33}
\end{array}\right)
$$




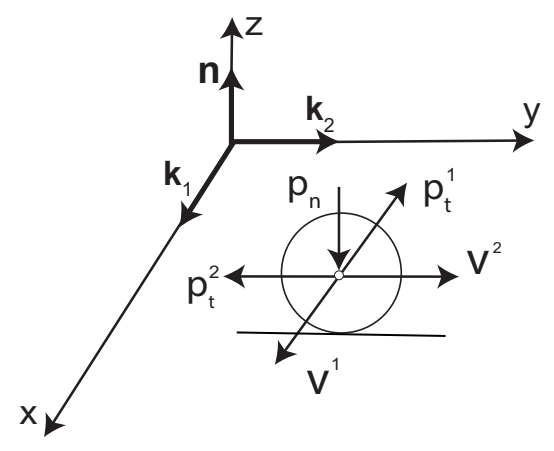

Figure 2: The planar sliding of the bead-like model of the macromolecule in presence of anisotropic dry friction in hypothetical base plane

In the case of horizontal orthotropy and transversal isotropy, the representation matrix of the viscous friction tensor $\mathbf{B}$ has four coefficients

$$
[\mathbf{B}]=\left(\begin{array}{ccc}
B^{11} & B^{12} & 0 \\
B^{12} & B^{22} & 0 \\
0 & 0 & B^{33}
\end{array}\right)
$$

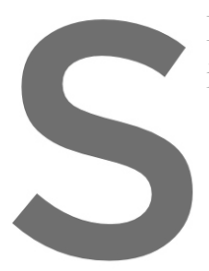

In the case of Cosserat-

is given by the following
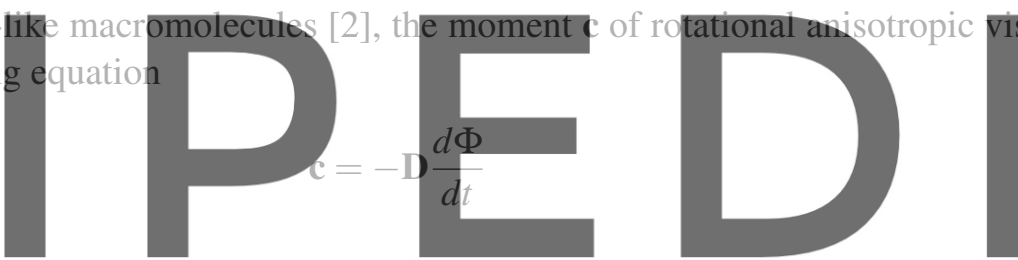

where, $\mathbb{D}$ is the tensor of rotational viscous friction

Register for free at https//Www.scipedia.com to download the version without the watermark

$$
\mathbb{D}=\mathbb{R}\left(\begin{array}{ccc}
D^{11} & 0 & 0 \\
0 & 0 & 0 \\
0 & 0 & 0
\end{array}\right) \mathbb{R}^{T}
$$

where, $\mathbf{R}=\mathbf{R}(\Phi)$ is the orthonormal rotation tensor, $D^{11}$ is the friction coefficient of the cylinder rotating about its own axis [2].

\subsection{Movements of the macromolecule models contacting with a hypothetical plane in presence of anisotropic dry friction}

During the motions the macromolecules are affected by surrounding molecules and friction forces arise. The frictional resistance between molecular chains must be considered with respect to the macromolecule model and the specific kinematics of the model.

Let us consider friction of the bead-like model of the macromolecule under sliding (Fig. 2). The bead model is dimensionless, due to this the friction forces are located at the bead center (Fig. 2). We assume 
orthotropic friction of the base plane, see Eq. (4), with two friction coefficients (for sliding along the axes $\mathrm{Ox}$ and $\mathrm{Oy}$ ). Components of the sliding velocity unit vector (Fig. 2) are as follows

$$
[\mathbf{v}]^{T}=\left[v^{1}, v^{2}\right]
$$

Then the components of the friction force vector (Fig. 2) are given by

$$
\left(\begin{array}{c}
p_{t}^{1} \\
p_{t}^{2}
\end{array}\right)=-\left|p_{n}\right|\left(\begin{array}{cc}
C^{11} & 0 \\
0 & C^{22}
\end{array}\right)\left(\begin{array}{l}
v^{1} \\
v^{2}
\end{array}\right)
$$

In the frame of the rod-like model we have considered the cylindrical rod rolling withou slipping due to a torque on the axle of the rod. The rod does not slip while rolling. There are conditions which must hold for rolling without slipping, i.e. the translational quantities depend on the angular quantities (distance, velocity and acceleration). The contact points of the rod touch the base plane with the relative velocity equal to zero. This is the stick case, the static friction force acts, see Eq. (1). We have analysed combined kinematics when the rod participate simultaneously in sliding and rolling motions.

In disc-like models of macromolecules we have considered spinning and sliding with respect to the base plane. Spinning is the angular motion about the normal axis to the plane. During spinning the disc particles contacting with the plane have translational velocities. Taking the linear friction model Eq. (4) or the nonlinear friction model Eq. (7) we can determine friction forces at the contact points. Additionally the moment of the friction force with respect to the center of spin can be determined. Using the integration the resultant friction force and the resultant friction moment are calculated. We have

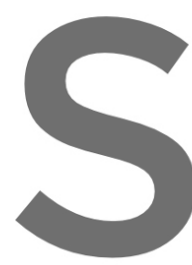
considered kinematics rigid discs in the presence In De Gennes tube model the friction anisotropy macromolecule. Due to this one should include direc Friction in the movement forwards is lower comp
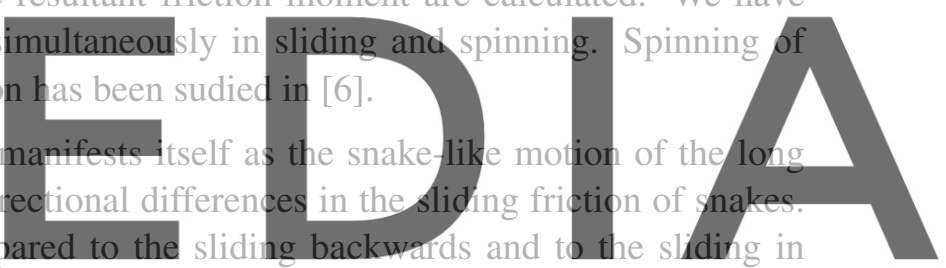

transverse directions. In this case we have used the non-centrosymmetric friction model, see Eq. (9)

Register fb; $\$$ ree at https//www.scipedia.com to download the version without the watermark

\section{CONCLUSIONS}

- Using numerical methods in contact mechanics and mechanical engineering every problem requires a proper constitutive relation not only for the material but also for friction. Therefore accurate friction constitutive models are needed in simulations of frictional properties of engineering materials (polymers).

- Proposed models of dry friction on surfaces of polymeric solids take into account: anisotropy, inhomogeneity and the evolution of surface microstructures. It is shown that the unit vector of the sliding velocity, the oriented angle and the curvature of the sliding trajectory are very useful in modelling of friction.

- The constitutive models of dry friction on surfaces of polymeric solids are based on phenomenological approaches with additional variables that describe microstructural effects. Constants of the friction force equations can be determined directly from experiments, or they can be defined with the aid of other material constants and operating conditions. 
- Proposed models of viscous and dry friction in the bulk polymeric materials describe friction anisotropy effects, and take into account micromechanical models of macromolecules. The friction descriptions are made in the frame of continuum-based and micromechanical approaches. The friction models in the bulk materials have practical applications in simulations of the polymer chain movements with friction and in predictiong of polymer behaviours.

- Nano-meter is the length scale of the polymer chain. Nano-second is the time scale in dynamics of polymer chains. Due to this, "trial and error method" can be a way of identification of the material parameters used in the micromechanical models.

- Polymeric solid-state materials have practical applications in self-lubricating bearings, mechanical seals, prostheses of human joints, vehicle tiries, etc. The research on self-lubricating polymers is important in view of a reduction or complete elimination of lubricants.

- Polymer melts, solutions, gels and complex fluids are used in various production processes of the chemical industry.

\section{REFERENCES}

[1] Briscoe, B.J. and Stolarski, T.A. The influence of contact zone kinematics on wear process of polymers. Wear (1991) 149(1-2):233-240.

[2] Cyron, C.J. and Wall, W.A. Numerical method for the simulation of the Brownian dynamics of rod like microstructures with three-dimensional nonlinear beam elements. Int. J. Num. Meth. Engng. (2012) 90(8):955-987.

[3] Dunn, A.C., Steffens, J.G., Burris, D.L., Banks, S.A. and Sawyer, W.G. Spatial geometric effects on the friction coefficients of UHMWPe. Wear (2008) 264(7-8):648-653.

[4] Liu, X.-X., Li,1T.-S., Liu, X.-J., Lv, R.-G. and Cong, P.-H. An investigation on the friction of oriented polytetrafluoroethylene (PTFE). Wear (2007) 262(11-12):1414-1418.

[5] Montesi, A., Morse, D.C. and Pasquali, M. Brownian dynamics algorithm for bead-rod semiflexible chain with anisotropic friction. J. Chem. Phys. (2005) 122(8):084903.

[6] Silantyeva, O. and Dmitriev, N. Motion of a thin eliptic plate under symmetric and asymmetric orthotropic friction forces. Surf. Topogr.: Metrol. Prop. (2018) 6 no. 1(015004):1-13.

[7] Zmitrowicz, A. A constitutive modelling of centrosymmetric and non-centrosymmetric anisotropic friction. Int. J. Solids Structurs. (1992) 29(23):3025-3043.

[8] Zmitrowicz, A. Illustrative examples of centrosymmetric and non-centrosymmetric anisotropic friction. Int. J. Solids Structurs. (1992) 29(23):3045-3059.

[9] Zmitrowicz, A. An equation of anisotropic friction with sliding path curvature effects. Int. J. Solids Structurs. (1999) 36(19):2825-2848.

[10] Zmitrowicz, A. Illustrative examples of anisotropic friction with sliding path curvature effects. Int. J. Solids Structurs. (1999) 36(19):2849-2863.

[11] Zmitrowicz, A. Models of kinematics dependent anisotropic and heterogeneous friction. Int. J. Solids Structurs. (2006) 43(14-15):4407-4451. 
[12] Zmitrowicz, A. Models of friction and wear of polymers: thermodynamic considerations. In: M. Raous (Ed.), Euromech No. 514, New Trends in Contact Mechanics, LMA CNRS Marseille (2012) pp.125-126. 